\title{
Soret effect on natural convection boundary-layer flow of a non- Newtonian nanofluid over a vertical cone embedded in a porous medium
}

\author{
F. M. Hady ${ }^{1}$, Mohamed R. Eid ${ }^{* 2}$, M. R. Abd-Elsalam ${ }^{3}$ and Mostafa A. Ahmed ${ }^{3}$ \\ ${ }^{1}$ Department of Mathematics, Faculty of Science, Assiut University, Assiut 71516, Egypt.) \\ ${ }^{2}$ Department of Science and Mathematics, Faculty of Education, Assiut University, The New Valley 72111, \\ Egypt. \\ ${ }^{3}$ Department of Mathematics, Faculty of Science, Sohag University, Sohag, Egypt .
}

\begin{abstract}
In this investigation, we intend to present the influence of the prominent Soret effect on natural convection boundary-layer flow of a non-Newtonian nanofluid over a vertical cone embedded in a porous medium. The model used for the nanofluid incorporates the effects of Brownian motion and thermophoresis. The governing partial differential equations are transformed into a set of non-linear equations and solved numerically using an efficient numerical shooting technique with a fourth-order Runge-Kutta scheme (MATLAB package). The numerical results for the temperature, volume fraction, and concentration profiles, as well as the reduced local Nusselt number $N u_{x} R a_{x}^{-1 / 2}$, the nanoparticle Sherwood number $N S h_{x} R a_{x}^{-1 / 2}$ and the regular Sherwood number $S h_{x} R a_{x}^{-1 / 2}$ are presented through plots which reveal interesting features. Comparisons with previously published work are performed and excellent agreement is obtained.
\end{abstract}

Keywords : natural convection, non-Newtonian nanofluid, porous medium, Soret effect.

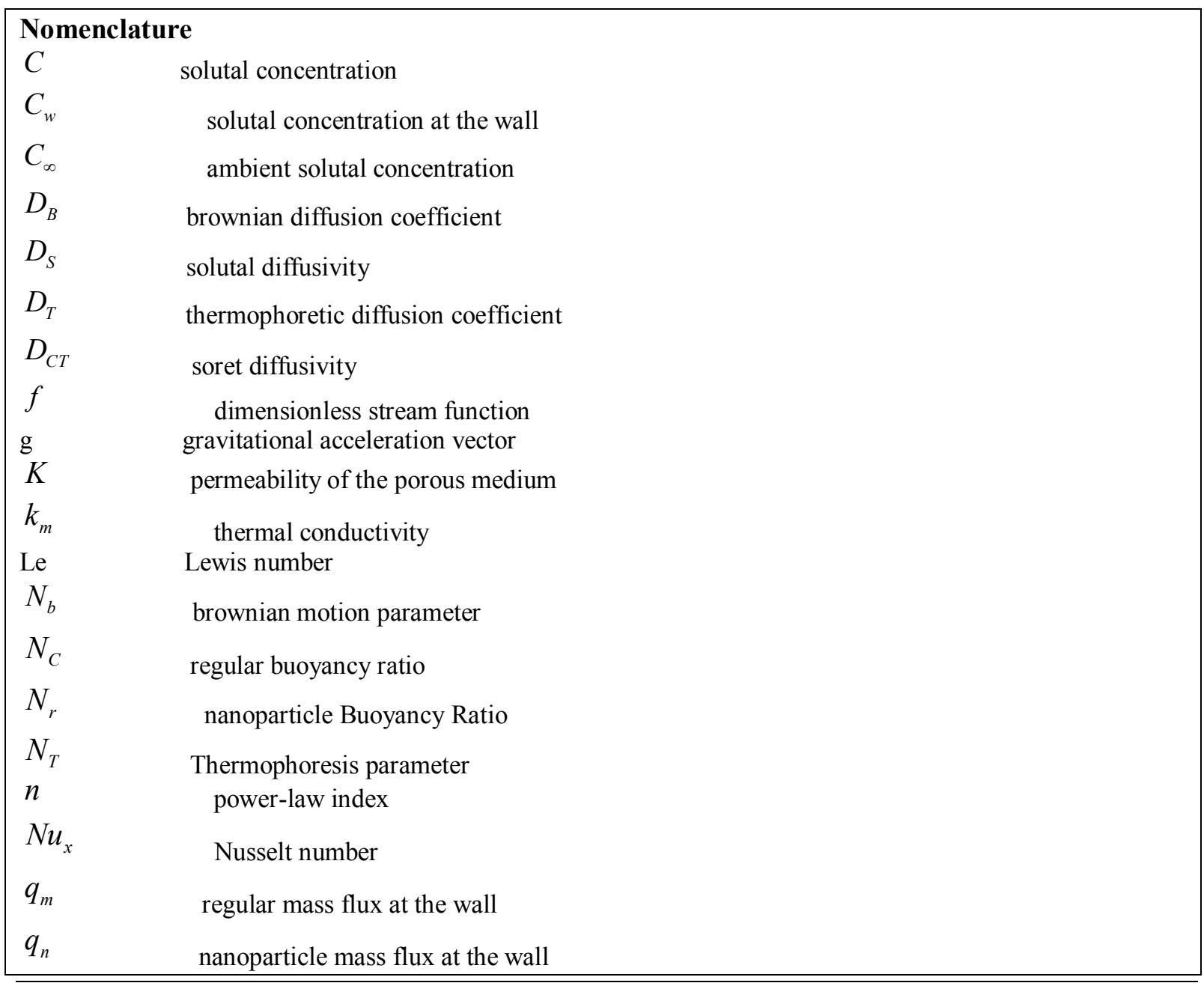




\begin{tabular}{|c|c|}
\hline$q_{w}$ & heat flux at the wall \\
\hline$R a_{x}$ & Local Rayleigh number \\
\hline$S$ & dimensionless concentration \\
\hline$S c$ & Schmidt number \\
\hline$S h_{x}$ & Local Sherwood number \\
\hline$N S h_{x}$ & Local nanoparticle Sherwood number \\
\hline$S r$ & Soret number \\
\hline$T$ & temperature \\
\hline$T_{w}$ & temperature at vertical plate \\
\hline$T_{\infty}$ & ambient temperature attained as y tends to infinity \\
\hline$u, v$ & velocity components along $\mathrm{x}$ - and $\mathrm{y}$-directions, respectively \\
\hline$v_{w}$ & uniform transpiration velocity \\
\hline$x, y$ & cartesian coordinates along the plate and normal to it, respectively \\
\hline Greek s & \\
\hline$\alpha$ & thermal diffusivity \\
\hline$\beta_{T}, \beta_{C}$ & volumetric thermal and solutal expansion coefficients of the base fluid. \\
\hline$\gamma$ & dimensionless volume fraction \\
\hline$\xi, \eta$ & similarity and non-similarity parameters \\
\hline$\theta$ & dimensionless temperature \\
\hline$\phi$ & nanoparticles volume fraction \\
\hline$\phi_{w}$ & nanoparticle volume fraction at the surface of the cone \\
\hline$\phi_{\infty}$ & ambient nanoparticle volume fraction attained as y tends to infinity \\
\hline$\lambda$ & half angle of the cone \\
\hline$\mu$ & fluid viscosity \\
\hline$\psi$ & stream function \\
\hline$\rho_{f}$ & fluid density \\
\hline$\rho_{P}$ & nanoparticle mass density \\
\hline$\left(\rho C_{p}\right)_{f}$ & heat capacity of the fluid \\
\hline$\left(\rho C_{p}\right)_{P}$ & effective heat capacity of the nanoparticles material \\
\hline Subscri & \\
\hline$C$ & concentration \\
\hline$T$ & temperature \\
\hline$w$ & condition at the wall \\
\hline$\infty$ & Stream function condition at the infinity \\
\hline Supersc & differentiation with respect to $\eta$ \\
\hline
\end{tabular}

\section{INTRODUCTION}

The study of convective heat transfer in nanofluids is gaining a lot of attention. The nanofluids have many applications in the industry since materials of nanometer size have unique physical and chemical properties. Nanofluids are solid-liquid composite materials consisting of solid nanoparticles or nanofibers with sizes typically of 1-100 nm suspended in liquid. Nanofluids have attracted great interest recently because of reports of greatly enhanced thermal properties. For example, a small amount $(<1 \%$ volume fraction) of $\mathrm{Cu}$ nanoparticles or carbon nanotubes dispersed in ethylene glycol or oil is reported to increase the inherently poor thermal conductivity of the liquid by $40 \%$ and $150 \%$, respectively as reported by Eastman et al. [1] and Choi et 
al. [2]. Conventional particle-liquid suspensions require high concentrations $(>10 \%)$ of particles to achieve such enhancement. However, problems of theology and stability are amplified at high concentrations, precluding the widespread use of conventional slurries as heat transfer fluids. In some cases, the observed enhancement in thermal conductivity of nanofluids is orders of magnitude larger than predicted by well-established theories. Nanofluids are used in different engineering applications such as microelectronics, microfluidics, transportation, biomedical, solid-state lighting and manufacturing. The research on heat and mass transfer in nanofluids has been receiving increased attention worldwide. Many researchers have found unexpected thermal properties of nanofluids, and have proposed new mechanisms behind the enhanced thermal properties of nanofluids. Excellent reviews on convective transport in nanofluids have been made by Buongiorno [3] and Kakac and Pramuanjaroenkij [4]. Kuznetsov and Nield [5] studied analytically the natural convective boundary-layer flow of a nanofluid past a vertical plate. The model used for the nanofluid incorporates the effects of Brownian motion and thermophoresis. Also, it is interesting to note that the Brownian motion of nanoparticles at molecular and nanoscale levels is a key nanoscale mechanism governing their thermal behaviors. In nanofluid systems, due to the size of the nanoparticles, the Brownian motion takes place, which can affect the heat transfer properties. As the particle size scale approaches to the nanometer scale, the particle Brownian motion and its effect on the surrounding liquids play an important role in the heat transfer.

The boundary-layer flow of non-Newtonian fluids in a porous media in which the main driving force is gravity has attracted a large class of applications in engineering practice, particularly in applied geophysics, geology, groundwater flow and oil reservoir engineering. Due to the increase in the production of heavy crude oils, and elsewhere where materials whose flow behavior in shear cannot be characterized by Newtonian relationships, it has become necessary to have an adequate understanding of the archeological effects of nonNewtonian fluid flows and, as a result, a new stage in the evolution of fluid dynamic theory is in progress. Chen and Chen [6] presented similarity solutions for free convection of non-Newtonian fluids over vertical surfaces in porous media. Mehta and Rao [7] investigated buoyancy induced flow of non-Newtonian fluids over a nonisothermal horizontal plate embedded in a porous medium. The effect of uniform lateral mass flux on natural convection about a cone embedded in a saturated porous medium is numerically analyzed by Yih [8]. Chamkha [9] considered coupled heat and mass transfer by mixed convection for a non-Newtonian power-law fluid flow over a permeable wedge embedded in a fluid-saturated porous medium. Nield and Kuznetsov [10] studied the natural convection past a vertical plate, in a porous medium saturated by a nanofluid. The model used for the nanofluid incorporates the effects of Brownian motion and thermophoresis.

The diffusion of mass due to temperature gradient is called Soret or thermo-diffusion effects ; this effect might become significant when density differences exist in the flow regime. For example, when species are introduced at a surface in fluid domain, with different (lower) density than the surrounding fluid Soret effect can be significant. The Soret parameter has been utilized for isotope separation and in mixture between gases with very light molecular weight $\left(\mathrm{H}_{2}, \mathrm{He}\right)$ and of medium molecular weight $\left(N_{2}\right.$, air). Dursunkaya and Worek[11] studied diffusion-thermo and thermal-diffusion effects in transient and steady natural convection from a vertical surface, whereas Kafoussias and Williams [12] presented the same effects on mixed convective and mass transfer transfer steady laminar boundary layer flow over a vertical flat plate with temperature dependent viscosity. The linear stability analysis of Soret-driven thermosolutal convection in a shallow horizontal layer of a porous medium subjected to inclined thermal and solutal gradients of finite magnitude was investigated theoretically by Narayana et al. [13]. Recently, the effect of melting and thermodiffusion on natural convection heat and mass transfer in a non-Newtonian fluid-saturated non-Darcy porous medium was presented by Kairi and Murthy [14].

Porous media heat transfer problems have several engineering applications such as geothermal energy recovery, crude oil extraction, ground water pollution, thermal energy storage and flow through filtering media. Cheng and Minkowycz [15] presented similarity solutions for free convective heat transfer from a vertical plate in a fluid saturated porous medium. Gorla and Zinolabedini [16] and Gorla and Tornabene [17] solved the nonsimilar problem of free convective heat transfer from a vertical plate embedded in a saturated porous medium with an arbitrarily varying surface temperature or heat flux. Chen and Chen [18] and Mehta and Rao [19] presented similarity solutions for free convection of non-Newtonian fluids over horizontal surfaces in porous media. Nakayama and Koyama [20] studied the natural convection over a non-isothermal body of arbitrary geometry placed in a porous medium. All these studies were concerned with Newtonian fluid flows. The boundary layer flows in nanofluids have been analyzed recently by Nield and Kuznetsov [10,21]. A clear picture about the nanofluid boundary layer flows is still to emerge. Rashad et al.[22] analyzed the effect of uniform transpiration velocity on free convection boundary layer flow of a non-Newtonian fluid over a permeable vertical cone embedded in a porous medium saturated with a nanofluid. The effects of Brownian motion and thermophoresis are included for the nanofluid. Hady et al. [23] reported the problem of non-Darcian free convection of a non-Newtonian fluid from a vertical sinusoidal wavy plate embedded in a porous medium. Hady and Ibrahim [24] studied the effect of the presence of an isotropic solid matrix on the forced convection 
heat transfer rate from a flat plate to power-law non-Newtonian fluid-saturated porous medium. Mahdy and Hady [25] studied the effects of thermophoretic particle deposition of the free convective flow over a flat plate embedded in non-Newtonian fluid-saturated porous medium in the presence of a magnetic field. The free convective heat transfer to the power-law non-Newtonian flow from a vertical plate in a porous medium saturated with nanofluid under laminar conditions is investigated by Hady et al. [26].

This study has been undertaken to analyze Soret effect on natural convection boundary-layer flow of a non-Newtonian nanofluid over a vertical cone embedded in a porous medium. The effects of Brownian motion and thermophoresis are included for the nanofluid. Numerical solutions of the boundary-layer equations are obtained and discussion is provided for several values of the nanofluid parameters governing the problem. The effects of buoyancy ratio, Soret number on the physical quantities of the flow, heat and mass transfer coefficients are analyzed. Comparison with previously published work is performed and the results are found to be in very good agreement.

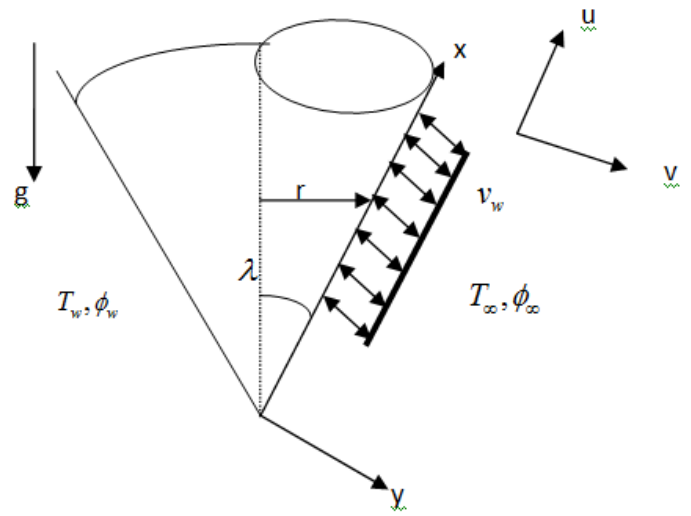

Fig. 1. Flow model and physical coordinate system.

\section{ANALYSIS}

Consider the problem of uniform transpiration velocity effect on free convection boundary-layer flow of a non-Newtonian fluid over a permeable vertical cone embedded in a porous medium saturated with a nanofluid. The model used for the nanofluid incorporates the effects of Brownian motionvand thermophoresis. It assumed that the cone surface is maintained at a constant temperature $T_{w}$, a constant nanoparticle volume fraction $\phi_{w}$ and a constant concentration $C_{w}$ and the ambient temperature, nanoparticle volume fraction and concentration far away from the surface of the cone $T_{\infty}, \phi_{\infty}$ and $C_{\infty}$ are assumed to be uniform. For $T_{w}>$ $T_{\infty}, \phi_{w}>\phi_{\infty}$ and $C_{w}>C_{\infty}$ an upward flow is induced as a result of the thermal and nanoparticle volume fraction buoyancy effects. Fig. 1 shows the flow model and physical coordinate system. The origin of the coordinate system is placed at the vertex of the cone, where $x$ and $y$ are Cartesian coordinates measuring distance along and normal to the surface of the cone, respectively. We adopt the model of non-Newtonian power-law fluid flow in a porous medium proposed by Christopher and Middleman [27] and Dharmadhikari and Kale [28]. The governing equations for the problem under consideration with the boundary-layer and Boussinesq approximations and the modified Darcy-law can be written as (see Yih [8]):

$$
\begin{aligned}
& \frac{\partial(r u)}{\partial x}+\frac{\partial(r v)}{\partial y}=0 \\
& \frac{\partial u^{n}}{\partial y}=\frac{\left(1-\phi_{\infty}\right) \rho_{f \infty} K g \cos \lambda}{\mu}\left[\beta_{T} \frac{\partial T}{\partial y}+\beta_{\mathrm{C}} \frac{\partial \mathrm{C}}{\partial y}\right]-\frac{\left(\rho_{\mathrm{P}}-\rho_{f \infty}\right) K g \cos \lambda}{\mu} \frac{\partial \phi}{\partial y}, \\
& u \frac{\partial T}{\partial x}+v \frac{\partial T}{\partial y}=\alpha \frac{\partial^{2} T}{\partial y^{2}}+\tau\left[D_{B} \frac{\partial \phi}{\partial y} \frac{\partial T}{\partial y}+\left(\frac{D_{T}}{T_{\infty}}\right)\left(\frac{\partial T}{\partial y}\right)^{2}\right] \\
& u \frac{\partial \phi}{\partial x}+v \frac{\partial \phi}{\partial y}=D_{B} \frac{\partial^{2} \phi}{\partial y^{2}}+\left(\frac{D_{T}}{T_{\infty}}\right) \frac{\partial^{2} T}{\partial y^{2}} \\
& u \frac{\partial C}{\partial x}+v \frac{\partial C}{\partial y}=D_{S} \frac{\partial^{2} C}{\partial y^{2}}+D_{C T} \frac{\partial^{2} T}{\partial y^{2}}
\end{aligned}
$$


where $x$ and $y$ denote the vertical and horizontal directions, respectively. $u, v, T, \phi$ and $C$ are the $x$ and $y$ components of velocity, temperature, nanoparticle volume fraction and concentration, respectively. $K, \beta_{T}, \beta_{C}, g, D_{B}, D_{T}, D_{S}$ and $D_{C T}$ are the permeability of the porous medium, volumetric thermal expansion coefficient of the nanofluid, volumetric solutal expansion coefficient of the nanofluid, gravitational acceleration vector, Brownian diffusion coefficient, thermophoretic diffusion coefficient, the solutal diffusivity and the Soret-type diffusivity, respectively. $\lambda, \mu, \rho_{f}$ and $\rho_{P}$ are the half angle of the cone, fluid viscosity, fluid density and nanoparticle mass density, respectively. $\alpha=k_{m} /(\rho C)_{f}$ and $\tau=(\rho C)_{P} /(\rho C)_{f}$ are the thermal diffusivity of porous medium and the ratio of heat capacities, respectively. $k_{m},(\rho C)_{f}$ and $(\rho C)_{P}$ are thermal conductivity, heat capacity of the fluid and the effective heat capacity of the nanoparticle material, respectively. We note that $\mathrm{n}<1$ and $>1$ represent pseudo-plastic fluid and dilatants fluid, respectively.

The boundary conditions suggested by the physics of the problem are given by

$$
\begin{aligned}
& v=v_{w} ; T=T_{w} ; \phi=\phi_{w} ; C=C_{w} \text { at } y=0 ; \\
& u=0 ; T \rightarrow T_{\infty} ; \phi \rightarrow \phi_{\infty} ; C \rightarrow C_{\infty} \text { as } y \rightarrow \infty,
\end{aligned}
$$

where $v_{w}, T_{\infty}$ and $\phi_{\infty}$ are the uniform transpiration velocity, temperature and nanoparticle volume fraction, respectively. We assumed the boundary layer to be sufficiently thin in comparison with the local radius of the cone. The local radius to a point in the boundary layer, therefore, can be replaced by the radius of the cone $r$, i.e., $r=x \sin \lambda$, invoking the following dimensionless variables. This can be done by introducing the stream function such that: $r u=\partial \psi / \partial y, r v=-\partial \psi / \partial x$ and using

$$
\begin{aligned}
& \xi=\frac{2 v_{w} x}{\alpha R a_{x}{ }^{1 / 2}}, \eta=\frac{y}{x} R a_{x}{ }^{1 / 2}, \quad f(\xi, \eta)=\frac{\psi(x, y)}{\alpha r R a_{x}^{1 / 2}}, \\
& \theta(\xi, \eta)=\frac{T-T_{\infty}}{T_{w}-T_{\infty}}, \gamma(\xi, \eta)=\frac{\phi-\phi_{\infty}}{\phi_{w}-\phi_{\infty}}, S(\xi, \eta)=\frac{C-C_{\infty}}{C_{w}-C_{\infty}} .
\end{aligned}
$$

Substituting Eq. (7) into Eqs. (1)-(5) produces the following non-similar equations:

$$
\begin{aligned}
& n f^{\prime \prime}\left(f^{\prime}\right)^{n-1}=\theta^{\prime}-N_{r} \gamma^{\prime}+N_{c} \mathrm{~S}^{\prime}, \\
& \theta^{\prime \prime}+N_{t} \theta^{\prime 2}+N_{b} \theta^{\prime} \gamma^{\prime}+\frac{3}{2} f \theta^{\prime}=\frac{1}{2} \xi\left[f^{\prime} \frac{\partial \theta}{\partial \xi}-\theta^{\prime} \frac{\partial f}{\partial \xi}\right], \\
& \gamma^{\prime \prime}+\frac{N_{t}}{N_{b}} \theta^{\prime \prime}+\frac{3 L e}{2} f \gamma^{\prime}=\frac{L e}{2} \xi\left[f^{\prime} \frac{\partial \gamma}{\partial \xi}-\gamma^{\prime} \frac{\partial f}{\partial \xi}\right], \\
& \frac{1}{S c} S^{\prime \prime}+S r \theta^{\prime \prime}+\frac{3}{2} f S^{\prime}=\frac{1}{2} \xi\left[f^{\prime} \frac{\partial S}{\partial \xi}-S^{\prime} \frac{\partial f}{\partial \xi}\right],
\end{aligned}
$$

With the boundary conditions:

$$
\begin{aligned}
& f(\xi, 0)=-\frac{\xi}{4}, \theta(\xi, 0)=1, \gamma(\xi, 0)=1, S(\xi, 0)=1, \\
& f^{\prime}(\xi, \infty)=0, \theta(\xi, \infty)=0, \gamma(\xi, \infty)=0, S(\xi, \infty)=0 .
\end{aligned}
$$

Where

$$
\begin{aligned}
& N_{c}=\frac{\beta_{\mathrm{C}}\left(C_{w}-\mathrm{C}_{\infty}\right)}{\beta_{T}\left(T_{w}-T_{\infty}\right)}, N_{r}=\frac{\left(\rho_{\mathrm{P}}-\rho_{f \infty}\right)}{\left(1-\phi_{\infty}\right) \rho_{f \infty}} \frac{\left(\phi_{w}-\phi_{\infty}\right)}{\beta_{T}\left(T_{w}-T_{\infty}\right)}, N_{b}=\frac{\tau}{\alpha} D_{B}\left(\phi_{w}-\phi_{\infty}\right), N_{t}=\frac{\tau}{\alpha}\left(\frac{D_{T}}{T_{\infty}}\right)\left(T_{w}-T_{\infty}\right), \\
& L e=\frac{\alpha}{D_{B}}, S c=\frac{\alpha}{D_{s}}, S r=\frac{D_{C T}}{\alpha} \frac{\left(T_{w}-T_{\infty}\right)}{\left(C_{w}-C_{\infty}\right)}, R a_{x}=\left(\frac{x}{\alpha}\right)\left[\frac{\left(1-\phi_{\infty}\right) K g \rho_{f \infty} \beta_{T} \cos \lambda\left(T_{w}-T_{\infty}\right)}{\mu}\right]^{\frac{1}{n}},
\end{aligned}
$$

are the regular buoyancy ratio, the nanofluid buoyancy ratio, Brownian motion parameter, thermophoresis parameter, Lewis number, Schmidt number, Soret number and modified local Rayleigh number, respectively. It 
should be noted that the mass flux parameter $\xi=0\left(v_{w}=0\right)$ corresponds to impermeable cone surface while $\xi>0\left(v_{w}>0\right)$ corresponds to the case of fluid injection and $\xi<0\left(v_{w}<0\right)$ corresponds to the case of fluid injection.

\subsection{HEAT AND MASS TRANSFER COEFFICIENTS}

The primary objective of this study is to estimate the parameters of engineering interest in fluid flow, heat and mass transport problems are the Nusselt number $N u$, the nanoparticles Sherwood number NSh, and the regular Sherwood number $S h$. These parameters characterize the surface drag, the wall heat, nanoparticle mass transfer and regular mass transfer rates, respectively. The local heat, local nanoparticle mass flux and local regular mass fluxes from the vertical plate can be obtained from:

$$
q_{w}=-k\left[\frac{\partial T}{\partial y}\right]_{y=0}, q_{n}=-D_{B}\left[\frac{\partial \phi}{\partial y}\right]_{y=0}, q_{m}=-D_{S}\left[\frac{\partial C}{\partial y}\right]_{y=0} .
$$

The Nusselt number $N u_{x}=\frac{q_{w} x}{k\left(T_{w}-T_{\infty}\right)}$, the nanoparticle Sherwood number $N S h_{x}=\frac{q_{n} x}{D_{B}\left(\phi_{w}-\phi_{\infty}\right)}$ and the regular Sherwood number $S h_{x}=\frac{q_{m} x}{D_{S}\left(C_{w}-C_{\infty}\right)}$, are given by:

$$
N u_{x}=-R a_{x}{ }^{1 / 2} \theta^{\prime}(\xi, 0), N S h_{x}=-R a_{x}{ }^{1 / 2} \gamma^{\prime}(\xi, 0), S h_{x}=-R a_{x}{ }^{1 / 2} S^{\prime}(\xi, 0) \text {. }
$$

Effect of the various parameters involved in the investigation on these coefficients is discussed in the following section.

\section{RESULTS AND DISCUSSION}

The non-linear Eqs. (8)-(11) are non-similar and possess no analytical solution and must be solved numerically subject to the boundary conditions given by Eq. (12). In this section, a representative set of graphical results for the temperature $\theta(\xi, \eta)$, nanoparticle volume fraction $\gamma(\xi, \eta)$ and concentration $S(\xi, \eta)$ as well as the reduced local Nusselt number $N u_{x}=-R a_{x}{ }^{1 / 2} \theta^{\prime}(\xi, 0)$ (reciprocal of rate of heat transfer), the nanoparticle Sherwood number $N S h_{x}=-R a_{x}{ }^{1 / 2} \gamma^{\prime}(\xi, 0)$ and the regular Sherwood number $S h_{x}=-R a_{x}{ }^{1 / 2} S^{\prime}(\xi, 0)$ are presented and discussed for various parametric conditions. These conditions are intended for various values of the viscosity index $n$, the Soret number $S r$, the Schmidt number $S c$, the regular buoyancy ratio $N_{c}$ and the mass flux parameter $\xi$ in the presence of the nanofluid buoyancy ratio $N_{r}$, Brownian motion parameter $N_{b}$, thermophoresis parameter $N_{t}$ and Lewis number $L e$. The accuracy of the aforementioned numerical method was validated by direct comparisons with the numerical results reported earlier by Rashad et al. [22] for various values of $n$ and $\xi$ in the absence of the nanoparticle volume fraction, Brownian motion, thermophoresis effects, the Soret number, Schmidt number and the regular buoyancy ratio $\left(N_{r}=N_{b}=N_{T}=S r=S c=N_{c}=0\right)$. This comparison is presented in Table 1. It can be shown from this table that an excellent agreement between the results exists.

To have a better understanding of the flow characteristics, numerical results for the temperature, nanoparticle volume fraction and concentration are calculated for different values of the parameters $\mathrm{Sr}, \mathrm{Sc}$, $N_{c}$ and $n$. Also, the effect of these parameters on non-dimensional heat, nanoparticle mass and regular mass transfer coefficients are discussed. The values of the remaining parameter are fixed.

Figs. 2(a)-2(c) show the non-dimensional temperature, volume fraction and concentration profiles for various values of Soret number $S r$ along with varying values of the viscosity index $n$, for a given $\left(N_{r}=0.5, N_{b}=0.3, N_{T}=0.1, S c=0.6, N_{c}=1.0, L e=10\right.$ and $\left.\xi=0.5\right)$ The Soret number $S r$ defines the effect of the temperature gradients inducing significant mass diffusion effects. It is noticed from Figs.(2) that an increase in the Soret number $S r$ resulted in an increase in the concentration fields while a decrease in the temperature and volume fraction is noted within the boundary layer. The present analysis shows that the flow field is appreciably influenced by the Soret parameter. It is clear from Figs.(2) that an increase in 
viscosity index $n$ increased the temperature, volume fraction and concentration. A reverse trend can be seen from Figs.(3) in the case of the Schmidt number $S c$, With the increase in the Schmidt number $S c$, a raise in the temperature and volume fraction whereas a reduction in the concentration field is seen. From Figs. 4(a)4(c) we observe that as the regular buoyancy ratio $N_{c}$ increase, the temperature, volume fraction and the concentration of the nanofluid decreased, but an increase in viscosity index $n$ increased the temperature, volume fraction and concentration of the nanofluid. Figs. 5(a)-5(c) present the effect of Soret number $S r$ and the viscosity index $n$ on the reduced local Nusselt number $-\theta^{\prime}(\xi, 0)$, the nanoparticle Sherwood number $-\gamma^{\prime}(\xi, 0)$ and the regular Sherwood number $-S^{\prime}(\xi, 0)$, respectively. From Figs. 5(a) and 5(b) we notice that as Soret number $S r$ increase, the reduced local Nusselt number $-\theta^{\prime}(\xi, 0)$ and the nanoparticle Sherwood number $-\gamma^{\prime}(\xi, 0)$ increases whereas the regular Sherwood number $-S^{\prime}(\xi, 0)$ decrease as shown by Fig. 5(c).Also, Figs. 5(a)-5(c) show the influence of viscosity index $n$ in the presence of the regular buoyancy ratio $N_{c}$, It can be seen that an increase in viscosity index $n$, it led to an decrease in the reduced local Nusselt number $-\theta^{\prime}(\xi, 0)$, the nanoparticle Sherwood number $-\gamma^{\prime}(\xi, 0)$ and the regular Sherwood number $-S^{\prime}(\xi, 0)$. Figs. 6(a) - 6(c) illustrate the effect of the Schmidt number $S c$ on the reduced local Nusselt number $-\theta^{\prime}(\xi, 0)$, the nanoparticle Sherwood number $-\gamma^{\prime}(\xi, 0)$ and the regular Sherwood number $-S^{\prime}(\xi, 0)$, respectively. As indicated before, increasing Schmidt number $S c$ causes increasing in the temperature profiles and volume fraction while the concentration decreases. This yields reduction in the local Nusselt number $-\theta^{\prime}(\xi, 0)$ and the nanoparticle Sherwood number $-\gamma^{\prime}(\xi, 0)$ and enhancement in the regular Sherwood number $-S^{\prime}(\xi, 0)$. As the regular buoyancy ratio $N_{c}$ increases, the reduced local Nusselt number $-\theta^{\prime}(\xi, 0)$, the nanoparticle Sherwood number $-\gamma^{\prime}(\xi, 0)$ and the regular Sherwood number $-S^{\prime}(\xi, 0)$ increase as shown by Fig. (7). Finally, Fig. (8) display the effects of viscosity index $n$ on the reduced local Nusselt number $-\theta^{\prime}(\xi, 0)$, the nanoparticle Sherwood number $-\gamma^{\prime}(\xi, 0)$ and the regular Sherwood number $-S^{\prime}(\xi, 0)$ in the presence of the regular buoyancy ratio $N_{c}=1.0$ and without the regular buoyancy ratio $N_{c}=0.0$. In the absence of the regular buoyancy ratio $N_{c}=0.0$, it is observed that the reduced local Nusselt number $-\theta^{\prime}(\xi, 0)$, the nanoparticle Sherwood number $-\gamma^{\prime}(\xi, 0)$ and the regular Sherwood number $-S^{\prime}(\xi, 0)$ increase with increases in viscosity index $n$, on the other hand, in the presence of the regular buoyancy ratio $N_{c}=1.0$, the reduced local Nusselt number $-\theta^{\prime}(\xi, 0)$, the nanoparticle Sherwood number $-\gamma^{\prime}(\xi, 0)$ and the regular Sherwood number $-S^{\prime}(\xi, 0)$ decrease with increases in viscosity index $n$.

\section{Figures AND TABLES}

Table 1

Values of $-\theta^{\prime}(\xi, 0)$ for various values of $\mathrm{n}$ in the absence of nanoparticle volume fraction, Brownian motion and thermophoresis effects $\left(\mathrm{N}_{\mathrm{r}}=\mathrm{N}_{\mathrm{b}}=\mathrm{N}_{\mathrm{t}}=N c=S r=S c=0\right)$.

\begin{tabular}{|c|c|c|}
\hline$N$ & Rashad et al.[22] & Present results \\
\hline 0.5 & 0.65225 & 0.6522 \\
0.8 & 0.73394 & 0.7339 \\
1.0 & 0.76865 & 0.7686 \\
1.1 & - & 0.7825 \\
1.2 & - & 0.7946 \\
\hline
\end{tabular}




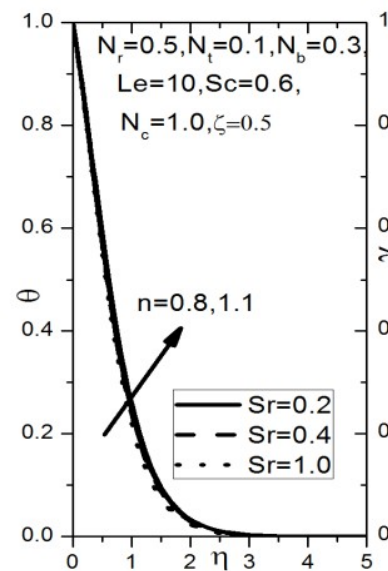

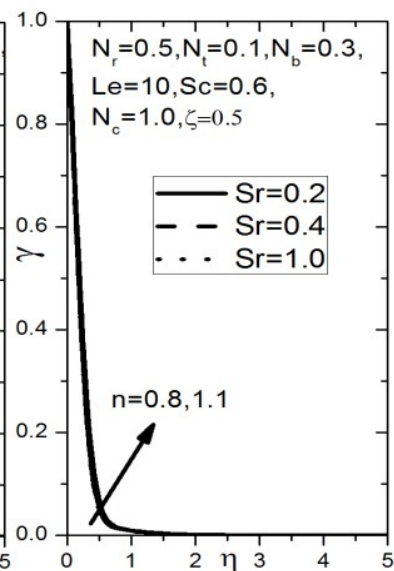

b

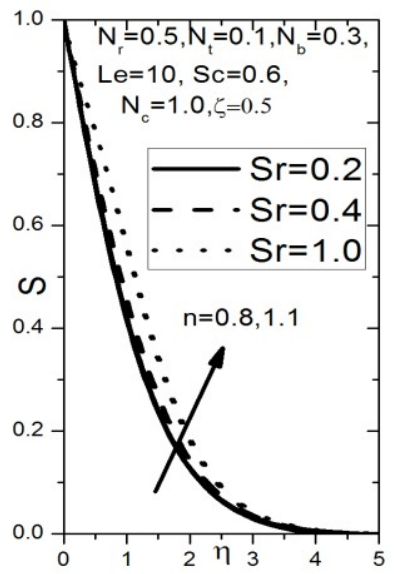

C

Fig.2.Effects of $S r$ and n on (a) Temperature, (b)volume fraction and (c)Concentration profiles.
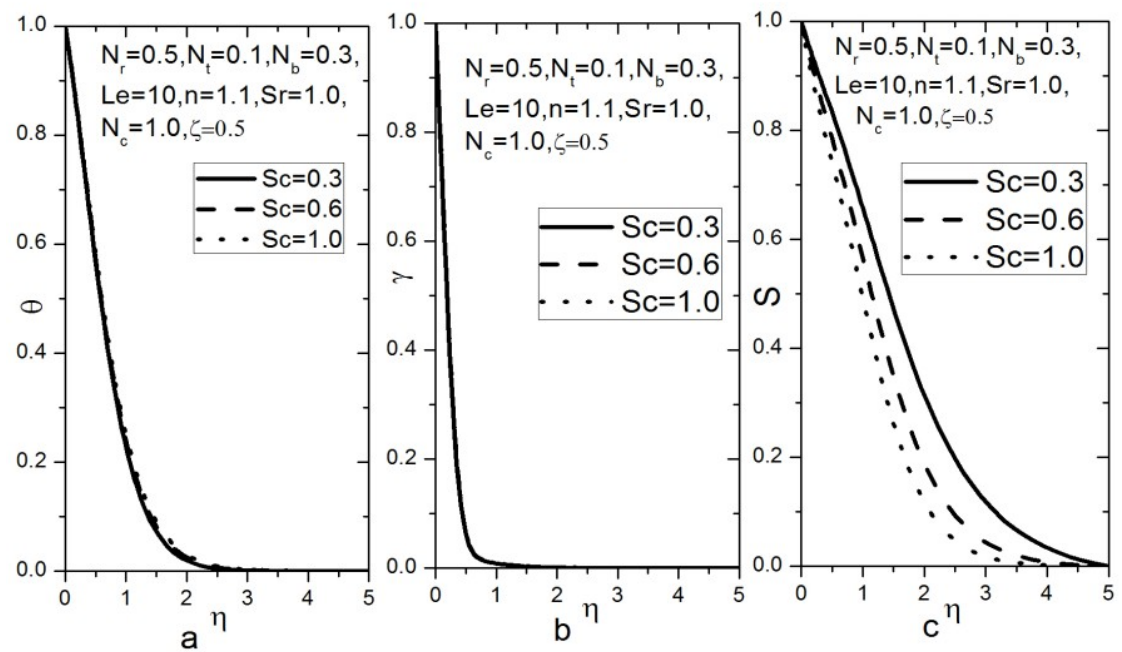

Fig.3. Effects of $S c$ on (a) Temperature, (b)volume fraction and (c)Concentration profiles.

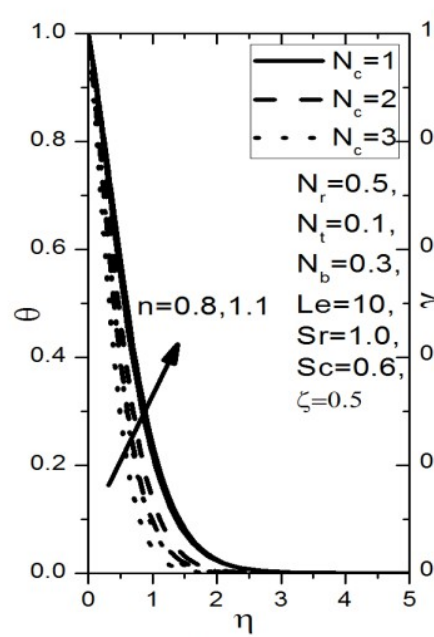

a

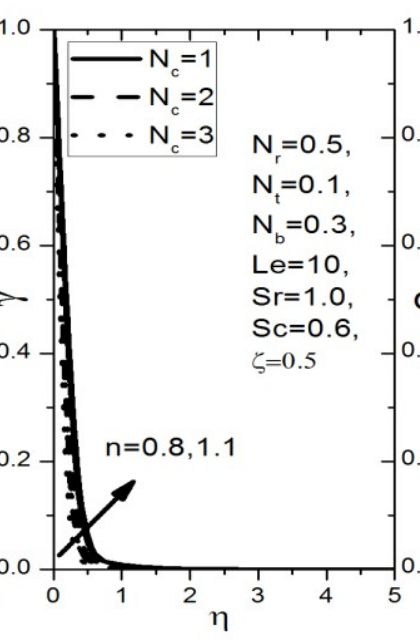

b

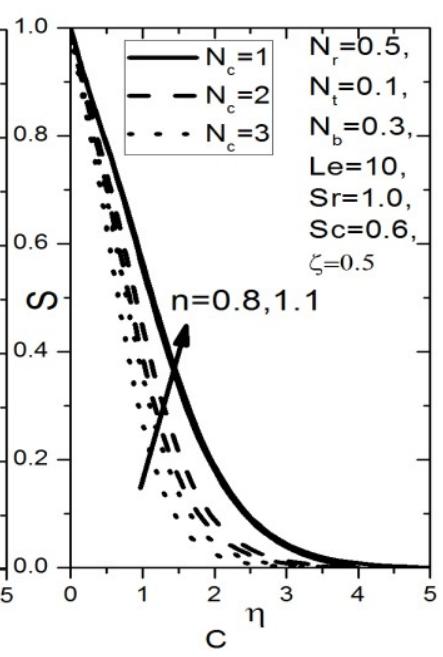

C

Fig.4. Effects of $N_{c}$ and $\mathrm{n}$ on (a) Temperature, (b)volume fraction and (c)Concentration profiles. 

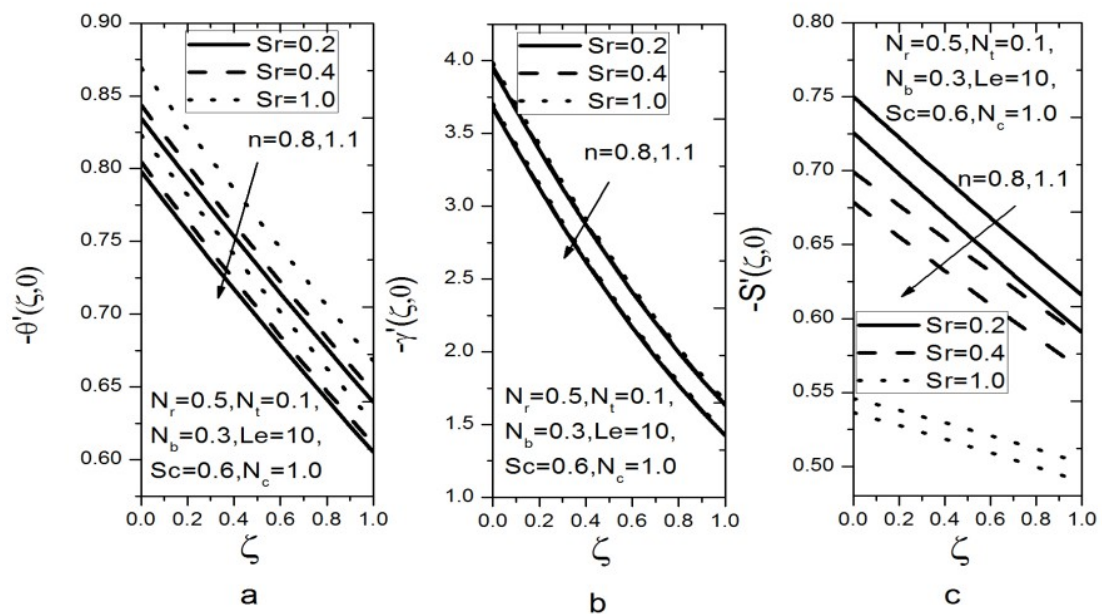

Fig.5. Effects of $S r$ and $\mathrm{n}$ on (a) the local Nusselt number, (b) the nanoparticle Sherwood number and (c)the regular Sherwood number.
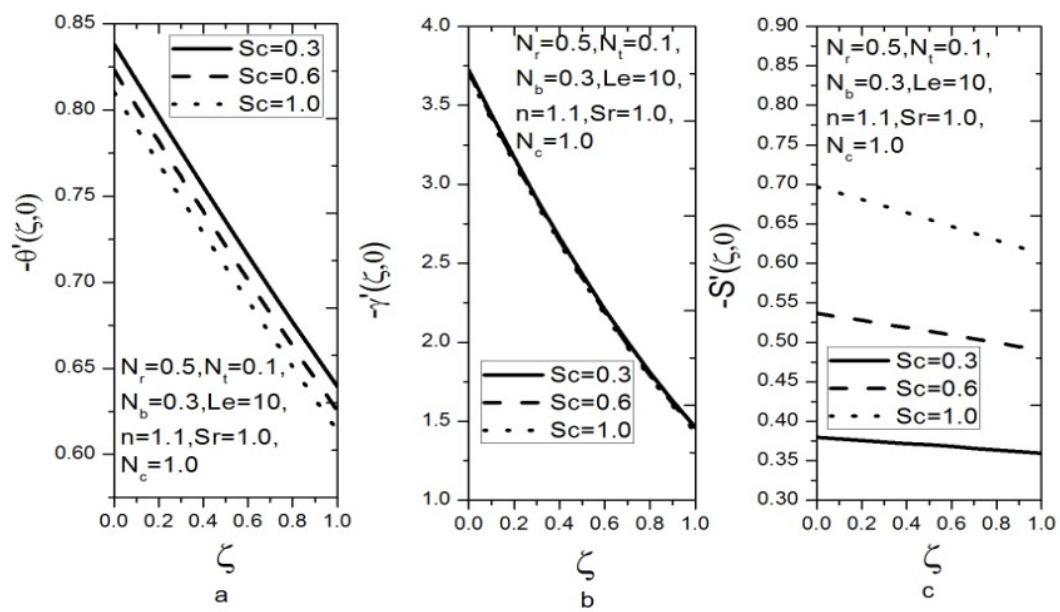

Fig.6. Effects of $S c$ on (a) the local Nusselt number, (b) the nanoparticle Sherwood number and (c)the regular Sherwood number.
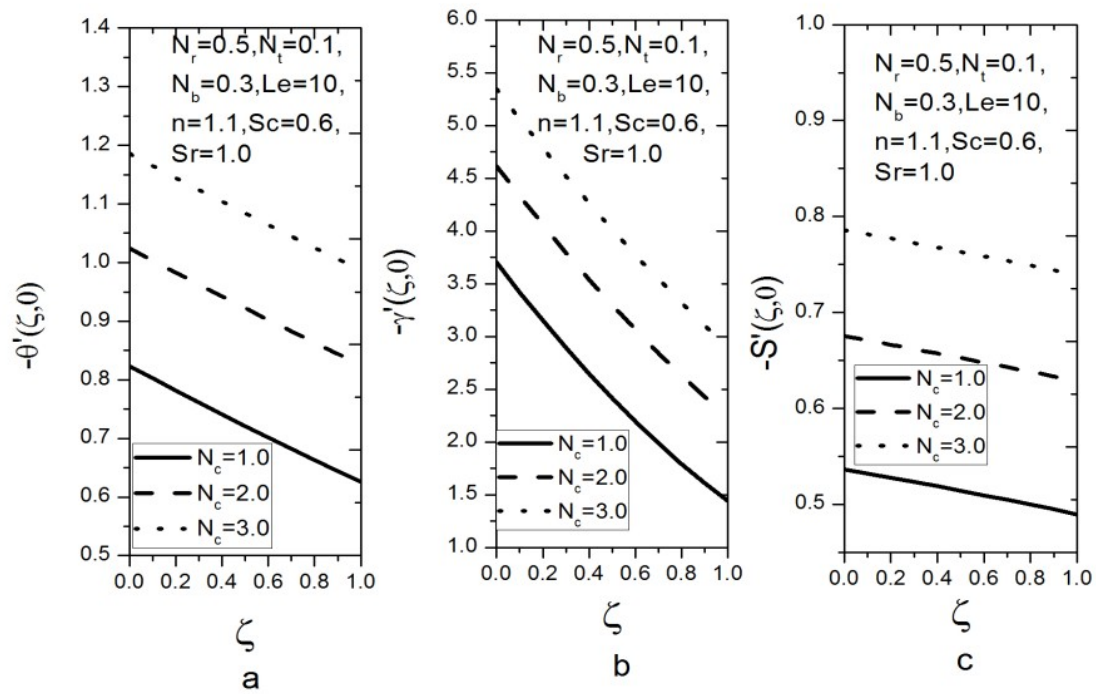

Fig.7. Effects of $N_{c}$ on (a) the local Nusselt number, (b) the nanoparticle Sherwood number and (c)the regular Sherwood number. 

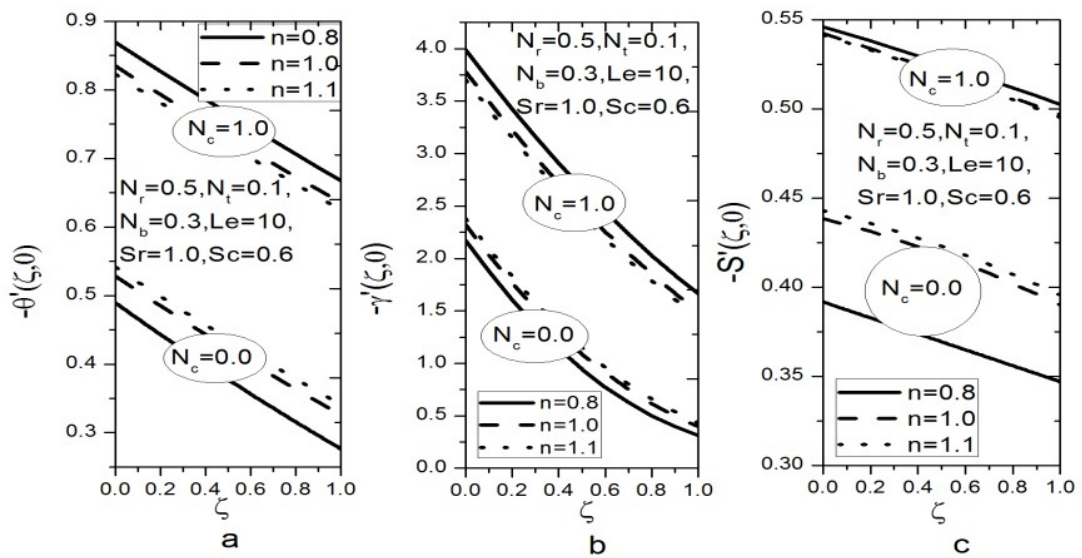

Fig.8. Effects of $N_{c}$ and $\mathrm{n}$ on (a) the local Nusselt number, (b) the nanoparticle Sherwood number and (c)the regular Sherwood number.

\section{CONCLUSION}

In the present work, we have studied theoretically the problem of Soret effect on natural convection boundary-layer flow of a non-Newtonian nanofluid over a vertical cone embedded in a porous medium. The model used for the nanofluid incorporates the effects of Brownian motion and thermophoresis. The obtained non-similar differential equations were solved numerically by using an efficient numerical shooting technique with a fourth-order Runge-Kutta scheme (MATLAB package). The results focused on the effects of the viscosity index $n$, the Soret number $S r$, the Schmidt number $S c$ and the regular buoyancy ratio $N_{c}$ on the reduced local Nusselt number $-\theta^{\prime}(\xi, 0)$, the nanoparticle Sherwood number $-\gamma^{\prime}(\xi, 0)$ and the regular Sherwood number $-S^{\prime}(\xi, 0)$ in the presence of the nanofluid buoyancy ratio $N_{r}$, Brownian motion parameter $N_{b}$, thermophoresis parameter $N_{T}$, Lewis number $L e$. The major conclusion is that the reduced local Nusselt number $-\theta^{\prime}(\xi, 0)$ and the nanoparticle Sherwood number $-\gamma^{\prime}(\xi, 0)$ increases whereas the regular Sherwood number $-S^{\prime}(\xi, 0)$ decrease, as Soret number $S r$ increase. Further, these quantities show reverse trend with the increasing values of Schmidt number $S c$. As the regular buoyancy ratio $N_{c}$ increases, the reduced local Nusselt number $-\theta^{\prime}(\xi, 0)$, the nanoparticle Sherwood number $-\gamma^{\prime}(\xi, 0)$ and the regular Sherwood number $-S^{\prime}(\xi, 0)$ increase. In the absence of the regular buoyancy ratio $N_{c}=0.0$, it is observed that the reduced local Nusselt number $-\theta^{\prime}(\xi, 0)$, the nanoparticle Sherwood number $-\gamma^{\prime}(\xi, 0)$ and the regular Sherwood number $-S^{\prime}(\xi, 0)$ increase with increases in viscosity index $n$, on the other hand, in the presence of the regular buoyancy ratio $N_{c}=1.0$, the reduced local Nusselt number $-\theta^{\prime}(\xi, 0)$, the nanoparticle Sherwood number $-\gamma^{\prime}(\xi, 0)$ and the regular Sherwood number $-S^{\prime}(\xi, 0)$ decrease with increases in viscosity index $n$.

\section{REFERENCES}

[1] J.A. Eastman, S.U.S. Choi, S. Li, W. Yu, L.J. Thompson, Anomalously Increased Effective Thermal Conductivities Containing Copper Nanoparticles, Applied Physics Letters 78 (2001) 718-720.

[2] S.U.S. Choi, Z.G. Zhang, W. Yu, F.E. Lockwood, E.A. Grulke, Anomalous Thermal Conductivity Enhancement on Nanotube Suspensions, Applied Physics Letters 79 (2001) 2252-2254.

[3] J. Buongiorno, Convective transport in nanofluids, ASME J Heat Transfer 128 (2006) 240-250.

[4] S. Kakac, A. Pramuanjaroenkij, Review of convective heat transfer enhancement with nanofluids, Int. J. Heat Mass Transfer 52 (2009) 3187-3196.

[5] A.V. Kuznetsov, D.A. Nield, Natural convective boundary-layer flow of a nanofluid past a vertical plate, Int. J. of Thermal Sciences 49 (2010) 243-247.

[6] H.T. Chen, C.K. Chen, Free convection of non-Newtonian fluids along a vertical plate embedded in a porous medium, Trans. ASME, J. Heat Transfer $110(1988) 257-260$.

[7] K.N. Mehta, K.N. Rao, Buoyancy-induced flow of non-Newtonian fluids over a non-isothermal horizontal plate embedded in a porous medium, Internat. J. Engrg. Sci. 32 (1994) 521-525.

[8] K.A. Yih, uniform lateral mass flux effect on natural convection of non-Newtonian fluids over a cone in porous media, Int. Commun. Heat Mass Transfer 25 (7) (1998) 959-968. 
[9] J. Ali Chamkha, Heat and mass transfer of a non-Newtonian fluid flow over a permeable wedge in porous media with variable wall temperature and concentration and heat source or sink, WSEAS Trans. Heat Mass Trans. 1 (5) (2010) 11-20.

[10] D.A. Nield, A.V. Kuznetsov, The Cheng-Minkowycz problem for natural convective boundary layer flow in a porous medium saturated by a nanofluid, Int. J. Heat Mass Transfer 52 (2009) 5792-5795.

[11] Z. Dursunkaya, W.M. Worek, Diffusion-thermo and thermal diffusion effects in transient and steady natural convection from a vertical surface, Int. J. Heat Mass Transfer 35 (1992) 2060-2065.

[12] N.G. Kafoussias, N.G. Williams, Thermal-diffusion and diffusion-thermo effects on mixed free-forced convective and mass transfer boundary layer flow with temperature dependent viscosity, Int. J. Engng. Sci. 33 (1995) 1369-1384.

[13] P.A.L. Narayana, P.V.S.N. Murthy, R.S.R. Gorla, Soret-driven thermo-solutal convection induced by inclined thermal and solutal gradients in a shallow horizontal layer of a porous medium, J. Fluid Mech. 612 (2008) 1-19.

[14] R.R. Kairi, P.V.S.N. Murthy, The effect of melting and thermo-diffusion on natural convection heat mass transfer in a non-Newtonian fluid saturated non-Darcy porous medium, Open Transp. Phenom. J. 1 (2009) 7-14.

[15] P. Cheng, W.J. Minkowycz, Free convection about a vertical flat plate embedded in a saturated porous medium with applications to heat transfer from a dike, J. Geophysics. Res. 82 (1977) 2040-2044.

[16] R.S.R. Gorla, A. Zinolabedini, Free Convection From a Vertical Plate With Nonuniform Surface Temperature and Embedded in a Porous Medium, Transactions of ASME, Journal of Energy Resources Technology 109 (1987) 26-30.

[17] R.S.R. Gorla, R. Tornabene, Free convection from a Vertical Plate With Nonuniform Surface Heat Flux and Embedded in a Porous Medium, Transport in Porous Media Journal 3 (1988) 95-106.

[18] H.T. Chen, C.K. Chen, Natural Convection of Non-Newtonian Fluids About a Horizontal Surface in a Porous Medium," Transactions of ASME, Journal of Energy Resources Technology 109 (1987) 119-123.

[19] K.N. Mehta, K.N. Rao, Buoyancy-induced Flow of Non-Newtonian Fluids in a Porous Medium Past a Horizontal Plate With Nonuniform Surface Heat Flux, International Journal of Engineering Science 32 (1994) 297-302.

[20] A. Nakayama, H. Koyama, Buoyancy-induced Flow of Non-Newtonian Fluids Over a Non-Isothermal Body of Arbitrary Shape in a Fluid-Saturated Porous Medium, Applied Scientific Research 48 (1991) 55-70.

[21] D.A. Nield, A.V. Kuznetsov, Thermal Instability in a Porous Medium Layer Saturated by a Nanofluid," International Journal of Heat and Mass Transfer 52 (2009) 5796- 5801.

[22] A.M. Rashad, M.A. EL-Hakiem, M.M.M. Abdou, Natural convection boundary layer of a non-Newtonian fluid about a permeable vertical cone embedded in a porous medium saturated with a nanofluid, Computers and Mathematics with Applications 62(8) (2011) 3140-3151.

[23] F. M. Hady, R. A. Mohamed, A. Mahdy, Non-Darcy natural convection flow along a vertical wavy plate embedded in a nonNewtonian fluid saturated porous medium. Int. J. Appl. Mech. Eng. 13 (2008) 91-100

[24] F. M. Hady, F. S. Ibrahim, Forced convection heat transfer on a flat plate embedded in porous media for power-law fluids. Trans. Porous Media 28 (1997) 125-134.

[25] A. Mahdy, F. M. Hady, Effect of thermophoretic particle deposition in non-Newtonian free convection flow over a vertical plate with magnetic field effect. J. Non-Newtonian Fluid Mech. 161 (2009) 37-41.

[26] F. M. Hady, F. S. Ibrahim, S. M. Abdel-Gaied, M. R. Eid, Boundary-layer non-Newtonian flow over vertical plate in porous medium saturated with nanofluid. Appl. Math. Mech. -Engl. Ed. 32(12) (2011) 1577-1586.

[27] R.H. Christopher, S. Middleman, Power-law flow through a packed tube, I \& EC Fundamentals, 4 (1965) $422-426$.

[28] R.V. Dharmadhikari, D. D. Kale, Flow of non-Newtonian fluids through porous media, Chem. Eng. Sci. 40 (1985) 527-529. 